\title{
A Raman study of Au/Te/Au/GaAs (100) ohmic contacts
}

H. Munder, C. Andrzejak, M. G. Berger, and H. Lüth

Institut für Schicht- und Ionentechnik, Forschungszentrum Jülich, P.O. Box 1913,

D-5170 Jülich, Germany

G. Borghs

Interuniversitair Micro Electronica Centrum, Kapeldreef 75, B-3001 Leuven, Belgium

K. Wuyts

K. U. Leuven, Physics Department, Instituut voor Kern en Stralingsfysika, Celestijnenlaan 200D,

B-3001 Leuven, Belgium

J. Watté and R. E. Silverans

K. U. Leuven, Physics Department, Laboratorium voor Vaste Stof-Fysika en Magnetisme, Celestijnenlaan 200D, B-3001, Leuven, Belgium

(Received 22 March 1991; accepted for publication 23 September 1991)

Ohmic contacts produced by high-energy pulsed laser beam alloying $\mathrm{Au} / \mathrm{Te} / \mathrm{Au} / n$-GaAs are investigated by micro Raman spectroscopy. The results are compared to those from furnace annealed ohmic contacts. For the furnace as well as for the laser annealed ohmic contacts, no evidence for a doping of the contact region is found in the Raman spectra. The presence of a highly disordered GaAs surface layer is observed for both types of contacts. In addition, after furnace processing $\mathrm{a} \mathrm{Ga}_{2} \mathrm{Te}_{3}$ layer is formed. These results are consistent with earlier Mössbauer studies. For the laser alloyed samples the results strengthen the role of a defective/disordered interface structure where conduction might occur by a resonant tunneling process involving localized gap states.

\section{INTRODUCTION}

The study of ohmic contacts to $n$-GaAs has been a main subject of research activities for many years. ${ }^{1}$ The most commonly used contact metallization is $(\mathrm{Ni} /) \mathrm{Au} /$ Ge which has become an industrial standard. ${ }^{2}$ Nowadays nonalloyed contacts like Pd/Ge (Ref. 3 ) become more important for the semiconductor technology. New developments which should also be mentioned are Pd/In (Ref. 4) and In/W (Ref. 5) contacts. However, the mechanism responsible for the ohmic behavior of especially the Ge based contacts is still a point of discussion.

The most popular model to explain the ohmic behavior for these contacts involves the formation of a highly doped GaAs surface layer. ${ }^{6}$ For good ohmic characteristics a doping level of about $2 \times 10^{19} \mathrm{~cm}^{-3}$ and a thickness of the doped layer of about $15 \mathrm{~nm}$ is required which allows quantum mechanical tunneling to occur due to the narrowing of the metal/semiconductor potential barrier. ${ }^{7}$ The second type of models requires the formation of a graded amorphous or crystalline heterojunction. ${ }^{8}$ In the model of a graded crystalline heterojunction the abrupt metal/GaAs transition is replaced by the formation of an intermediate mixed semiconductor layer. The composition of this layer varies in such a way that the potential barrier is reduced. The required grading width depends on the doping level of the substrate and of the overlayer. For an amorphous heterojunction the current transport can be due to a phonon assisted hopping or alternatively by a multistep tunneling process between localized gap states at the metal/ semiconductor interface.

To extend the study of the nature of $n$-GaAs ohmic contacts, a Au/Te metallization scheme, which-after fur- nace or laser alloying-results in specific contact resistivities comparable to those of the $\mathrm{Au} / \mathrm{Ge}$ contacts was developed. ${ }^{9}$ Foregoing studies of these contacts by Mössbauer spectroscopy and $x$-ray diffraction (XRD), ${ }^{10,11} \mathrm{Ru}$ therford backscattering (RBS), and Auger electron spectroscopy (AES), ${ }^{12}$ are marked by the absence of any evidence in favor of the doping model, whereas strong arguments for both the graded crystalline and amorphous heterojunction model were obtained. However, although Mössbauer spectroscopy has proven to be sensitive in probing the substitution of $\mathrm{Te}$ dopants on electrically active sites (substitutional on As site), ${ }^{13}$ its sensitivity is limited to Te configurations representing more than 1 percent of the total. Therefore the presence of a thin highly doped $n^{+}+$layer could not be ruled out. On the other hand Raman spectroscopy is a very sensitive method in probing the doping of semiconductors. Doping levels $\geqslant 10^{17} \mathrm{~cm}^{-3}$ can easily be detected even if the width of the doped layer is very small.

In this letter we report Raman spectroscopical investigations of both furnace and pulsed laser alloyed $\mathrm{Au} / \mathrm{Te} / \mathrm{Au} / \mathrm{GaAs}$ contacts. To identify the phases after alloying, $\mathrm{Ga}_{2} \mathrm{Te}_{3}$ and $\mathrm{Te}$ single crystals have been studied as well. Also $n^{+}+$GaAs layers with thicknesses between 17 and $230 \mathrm{~nm}$ grown on semi-insulating (SI) and $n$-doped $\left(n=3 \times 10^{17} \mathrm{~cm}^{-3}\right)$ substrates are investigated. The results of resistance measurements on the contacts are briefly summarized.

\section{EXPERIMENTAL}

The metallization was performed by successive deposition of $50 \AA \mathrm{Au}, 500 \AA \mathrm{Te}$ and $1200 \AA \mathrm{Au}$ on (100) 


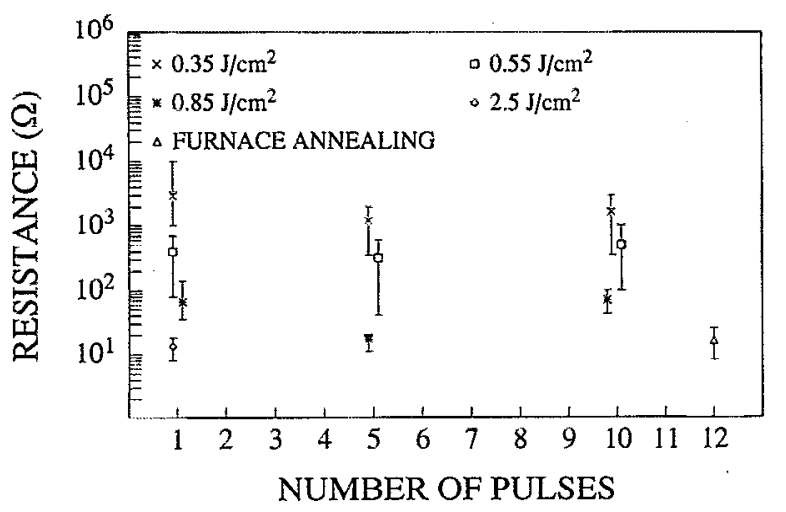

FIG. 1. Resistance values deduced from $I-V$ measurements for pulsed laser mixed $\mathrm{Au} / \mathrm{Te} / \mathrm{Au} / n$-GaAs contacts, as a function of energy fluency and number of pulses. For comparison, the contact resistance on furnace annealed samples $\left(T_{\text {alloy }}>500^{\circ} \mathrm{C}\right)$ is shown.

oriented GaAs. For the pulsed laser beam mixing a Nd:YAG laser $(\lambda-532 \mathrm{~nm}, t=7 \mathrm{~ns})$ was used. ${ }^{10}$ Different contact structures were produced by varying the laser energy fluency and the number of pulses. The GaAs substrate material for Raman investigations was semi-insulating for the laser beam mixed samples and semi-insulating and uniformly ( $\mathrm{Si}$ ) doped $\left(n=3 \times 10^{17} \mathrm{~cm}^{-3}\right)$ for the furnace alloyed samples. The furnace alloying was performed in a graphite strip heater. The samples were heated for $15 \mathrm{~s}$ under a forming gas ambient.

The resistance valucs determined by $I-V$ measurements on samples with uniformly doped substrate material, are shown in Fig. 1 for laser mixed samples with different treatments (see also Ref. 10). Ohmic contacts are those with a resistance $<25 \Omega .{ }^{10,12}$ The laser annealed contacts show a reduction in the resistance by increasing the energy fluency of a single pulse or by increasing the number of pulses. Contact resistances comparable to those of furnace annealed samples $\left(T_{\text {alloy }} \leqslant 500^{\circ} \mathrm{C}\right)$ have been achieved.

Raman measurements were made at room temperature in backscattering configuration, using the 457,488 , and $514 \mathrm{~nm}$ lines of an $\mathrm{Ar}^{+}$ion laser and the $647 \mathrm{~nm}$ line of a $\mathrm{Kr}^{+}$ion laser. The information depth in Raman measurements is given by the light penetration depth which varies between 50 and $200 \mathrm{~nm}$ for the different laser lines in GaAs. The scattered light was analyzed by a Dilor XY triple monochromator with multichannel detection which allowed micro and macro Raman experiments. Thereby the spatial resolution was about 2 and $100 \mu \mathrm{m}$, respectively. The power of the incident laser light was kept below $1 \mathrm{~mW}$ to protect the samples against heating effects during the Raman measurements. In these contact structures gallium telluride compounds were the materials most sensitive against overheating. Hence a laser power of $10 \mathrm{~mW}$ was only used if no gallium telluride features were observable: To exclude any heating effects macro Raman measurements with a laser power of $0.2 \mathrm{~mW}$ were performed.

In Raman spectra information about the structure and the composition of the samples is given by the phonon frequencies, while information about the doping level can

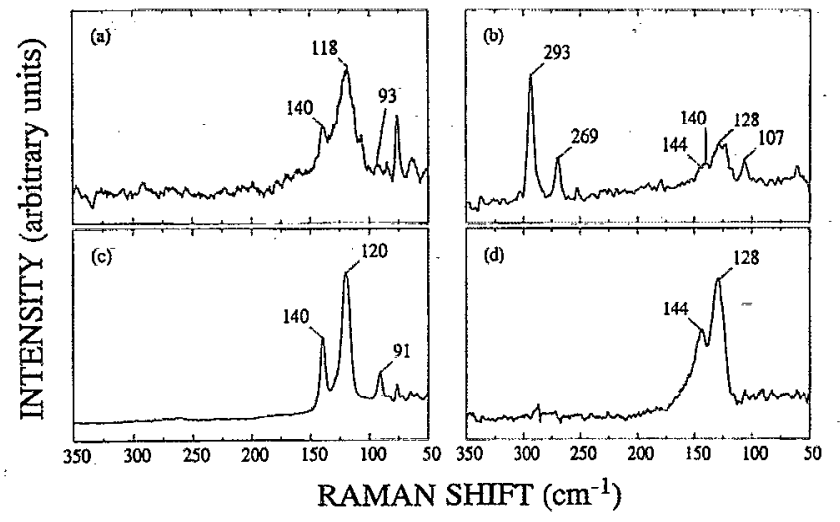

FIG. 2. (a) (b) Raman spectra of ohmic contacts produced by furnace annealing $\left(\lambda_{L}=457 \mathrm{~nm}\right)$. (c): Raman spectrum of a Te single crystal $\left(\lambda_{L}=514 \mathrm{~nm}\right)$. (d): Raman spectrum of a $\mathrm{Ga}_{2} \mathrm{Te}_{3}$ single crystal $\left(\lambda_{i}=514 \mathrm{~nm}\right)$.

be obtained from the frequency of coupled plasmon LO phonon modes. ${ }^{14.15}$ Therefore, Raman measurcments give information about the mechanisms responsible for the ohmic character of laser and furnace alloyed contacts. The two different contributions (doping and structure) to the spectra have different symmetry selection rules. The symmetry can be probed by using different orientations for the polarizations of the incident and scattered light. For example, for a backscattering experiment from a (100) surface with the polarization of the incident light along (010) and for the scattered light along (001) dircetion of the substrate the scattering configuration will in the following be indicated by: $z(x y) \bar{z}$.

\section{RESULTS AND DISCUSSION}

First we discuss the furnace annealed ohmic contacts. In Fig. 2 Raman spectra are shown which have been obtained with a power of the incident laser light $\leqslant 1 \mathrm{~mW}$. By probing different positions on the sample surface an inhomogeneity of the sample is found. From some parts of the sample no Raman signals are detected. This can be explained by the presence of metal islands composed of $\mathrm{Au}$ and $\mathrm{Ga}$ which are indicated by transmission electron microscopy (TEM) and AES results. ${ }^{12,16}$ In between the islands different features in the Raman spectra are observed [Figs. 2(a) and 2(b)]. In Fig. 2(a) there are peaks at 140, 118 , and $93 \mathrm{~cm}^{-1}$ which correspond to the phonon frequencies of Te [Fig. 2(c)]. The larger full width at half maximum (FWHM) of the phonon peaks in Fig. 2(a) is due to a polycrystalline morphology of the Te. Additional peaks at 128 and $145 \mathrm{~cm}^{-1}$ are found. By comparing Fig. 2(b) to a spectrum taken from a $\mathrm{Ga}_{2} \mathrm{Te}_{3}$ single crystal [Fig. 2(d)] the formation of $\mathrm{Ga}_{2} \mathrm{Te}_{3}$ can be assumed. The peak at $119 \mathrm{~cm}^{-1}$ in Fig. 2(b) is due to the coexistence of $\mathrm{Te}$ at this location. The nature of the peak at $107 \mathrm{~cm}^{-1}$ is not yet understood. From macro Raman experiments heating effects during the measurements can be ruled out.

Also signals from the GaAs region below the ohmic contact [Fig. 2(b)] are detected. A part from the symmetry 


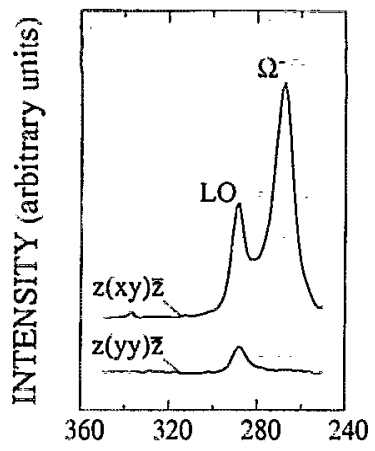

(a) RAMAN SHIFT $\left(\mathrm{cm}^{-1}\right)$

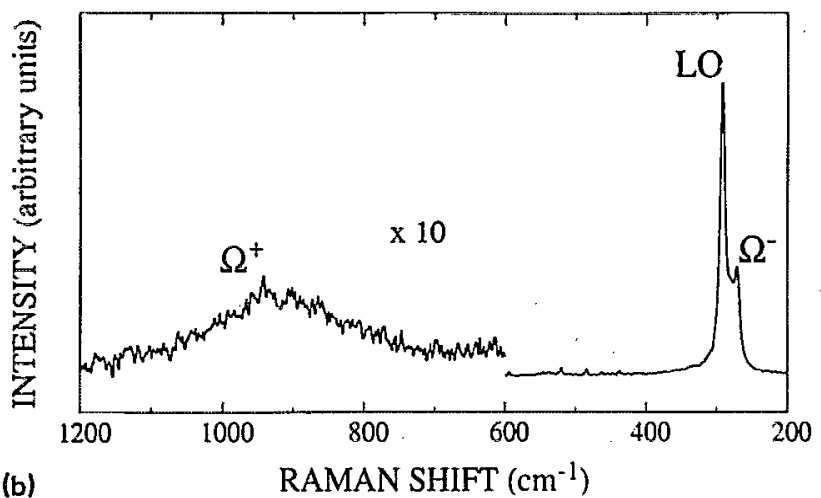

FIG. 3. Spectra from heavily doped reference samples grown by MBE $\left(\lambda_{L}=457 \mathrm{~nm}\right)$. (a): Light scattering from an $\Omega^{-}$mode using different scattering configurations. The thickness of the doped layer is $230 \mathrm{~nm}$ and the doping level is $1.3 \times 10^{19} \mathrm{~cm}^{-3}$. (b): Spectrum from a heavily doped GaAs sample with a thickness of the doped layer of $30 \mathrm{~nm}$. The frequency of the $\Omega^{+}$mode corresponds to a doping level of $8 \times 10^{18} \mathrm{~cm}^{-3}$.

allowed LO phonon at $291 \mathrm{~cm}^{-1}$, a peak at $269 \mathrm{~cm}^{-1}$ is observed. There are two possible explanations for the appearance of this peak. First it can be an $\Omega^{-}$mode of coupled plasmon LO phonon modes (PLP) which are observed in doped GaAs. ${ }^{14,15}$ Secondly this peak can be explained by the existence of a high density of defects. This will relax the selection rules yielding $\mathbf{k}$-induced scattering processes from TO phonons. k-induced scattering occurs in highly disordered samples like ion implanted ${ }^{17}$ or ion bombarded ${ }^{18} \mathrm{GaAs}$ samples.

To distinguish between these two possible explanations heavily doped $\mathrm{GaAs}$ reference samples are studied, spectra are shown in Fig. 3. In doped GaAs the fluctuations in the free carrier density and the LO phonon interact via their electric fields leading to coupled PLP modes. ${ }^{14,15}$ The frequencies of these modes ( $\Omega^{-}$and $\Omega^{+}$mode) allow the estimation of doping levels in between $10^{16}$ and $10^{20} \mathrm{~cm}^{-3}$. Depending on their frequencies the character is more phonon or plasmon like, leading to different symmetry selection rules. Especially an $\Omega^{-}$mode close to the TO phonon frequency of GaAs has the same symmetry as the LO phonon and will give only a contribution to the spectra in the allowed scattering configuration $z(x y) \bar{z}$ [Fig. 3(a)].

To find out how sensitive Raman spectroscopy is for the proof of the presence of doped layers very thin heavily doped $\left(n \approx 8 \times 10^{18} \mathrm{~cm}^{-3}\right)$ molecular beam epitaxy

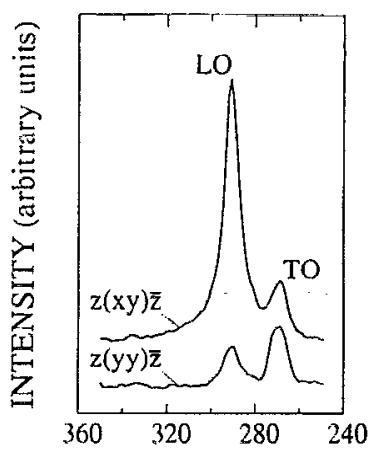

FIG. 4. The peak at $269 \mathrm{~cm}^{-1}$ in spectra obtained on ohmic contacts is a disorder activated TO phonon $\left(\lambda_{L}=457\right.$ $\mathrm{nm})$. Its intensity is the same for both scattering configurations (sample: $n=3 \times 10^{17} \mathrm{~cm}^{-3}, T_{\text {alloy }}=550^{\circ} \mathrm{C}$ ).
RAMAN SHIFT $\left(\mathrm{cm}^{-1}\right)$
(MBE) grown samples are studied. The spectrum shown in Fig. 3(b) was taken on a sample with a thickness of the doped layer equal $30 \mathrm{~nm}$. However from the fact that the $\Omega^{+}$mode can clearly be observed on samples where the thickness of the doped layer is $17 \mathrm{~nm}$, the limit for the width of the doped layer which is detectable should be below $15 \mathrm{~nm}$. Obviously the detection limit must be larger than the sum of the width of the depletion layers at the surface and the interface, because for measuring PLP modes fluctuations in the free carrier density are necessary. Therefore, for higher doping levels the presence of doped layers with thicknesses even smaller than $15 \mathrm{~nm}$ can be detected. For these Raman studies the 457-nm laser line has been used, because the light penetration depth must be larger than the width of the surface depletion layer. That is the reason why the intensity and therefore the sensitivity could not be enhanced by using a laser line $(413 \mathrm{~nm})$ which is in resonance to one of the energy gaps of GaAs.

Taking these results into account an explanation of the $269 \mathrm{~cm}^{-1}$ peak, which has been observed in the spectra of the ohmic contacts can be given. If this peak is assumed to be an $\Omega^{-}$PLP mode, then an $\Omega^{+}$mode must occur at higher wave numbers. However we do not observe such a peak even if a laser line $\left(\lambda_{L}=647 \mathrm{~nm}\right)$ with a larger penetration depth is used. Beside this, the intensity of the 269 $\mathrm{cm}^{-1}$ peak has been the same for both scattering configurations $z(x y) \bar{z}$ and $z(y y) \bar{z}$ (Fig. 4). From these facts the explanation by a PLP mode can be excluded and the peak can only be explained by a disorder activated $\mathbf{k}$-induced TO phonon scattering.

Thus we can conclude, that the ohmic behavior of the furnace annealed contacts is due to a heterojunction between disordered $\mathrm{GaAs}$ and $\mathrm{Ga}_{2} \mathrm{Te}_{3}$. These results are compatible with former Mössbauer and RBS measurements. ${ }^{11,12}$ An explanation of the ohmic characteristic based on $n^{+}$doping of the $\mathrm{Ga}_{2} \mathrm{Te}_{3}$ layer caused by diffusion of As remains possible, but could not be studied by Raman spectroscopy. ${ }^{19}$

In the Raman spectra from the laser annealed contacts different features are observed, depending on the energy fluency and the number of pulses during the annealing process. In Fig. 5, the Raman spectra of a highly resistive contact $\left(0.35 \mathrm{~J} / \mathrm{cm}^{2}, 5\right.$ pulses $)$ which show a lateral inhomogeneity are presented. Beside other peaks a broad structure around $130 \mathrm{~cm}^{-1}$ is observed [Fig. 5(a)]. Comparing 

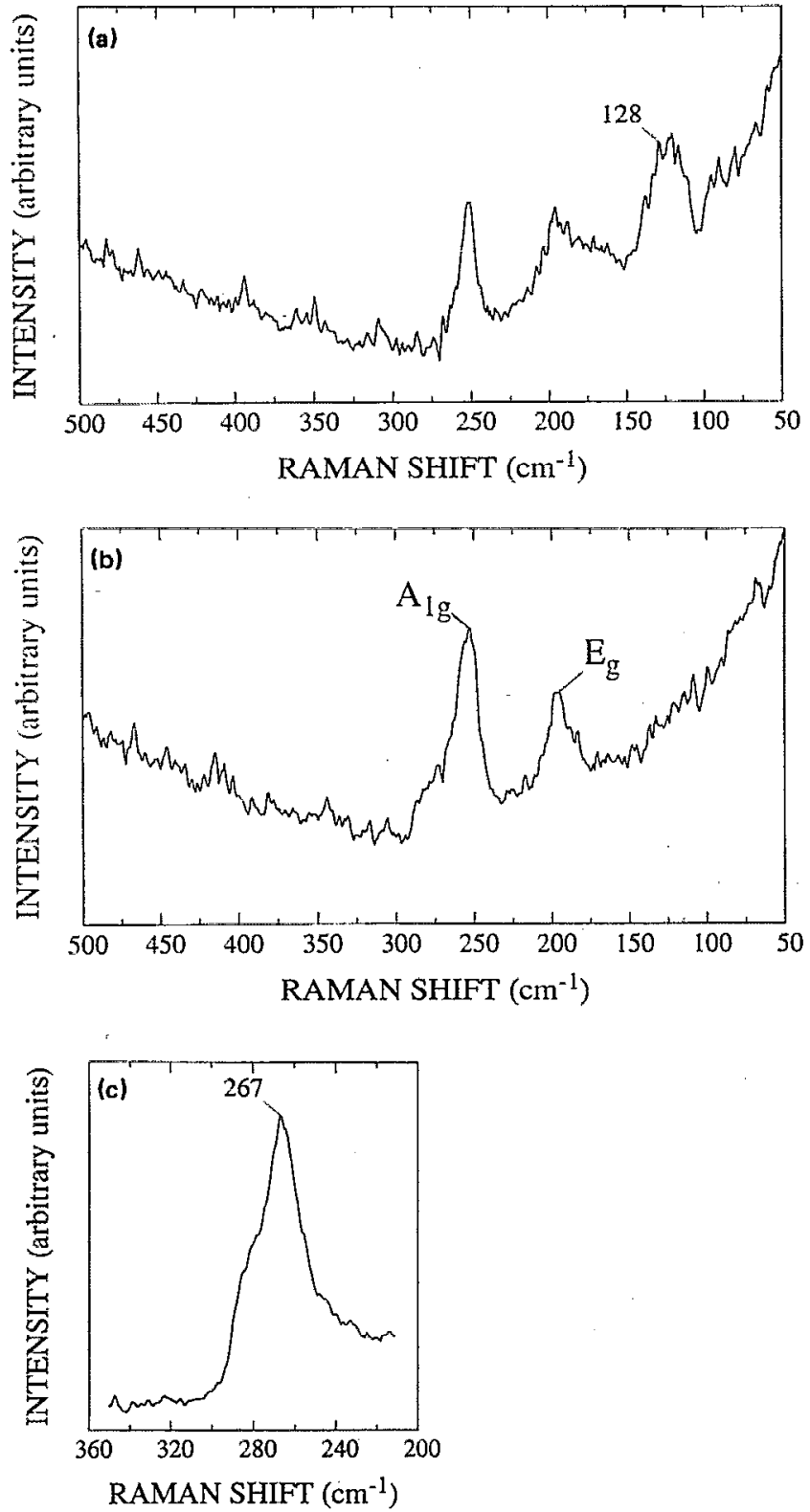

FIG. 5. Raman spectra from laser annealed contacts $\left(\lambda_{L}=514 \mathrm{~nm}\right)$. The nonohmic samples $\left(0.35 \mathrm{~J} / \mathrm{cm}^{2}, 5\right.$ pulses $)$ show a lateral inhomogeneity ( $a$ and $b$ ) and on ohmic samples $\left(0.85 \mathrm{~J} / \mathrm{cm}^{2}, 5\right.$ pulses) amorphouslike GaAs (c) is found. (a) Gallium telluride features around $130 \mathrm{~cm}^{-1}$, (b) $A_{1 \mathrm{~g}}$ and $E_{g}$ of As, (c) amorphous-like GaAs.

this with literature data scattering from amorphous ${ }^{20}$ and crystalline Te can be ruled out. However, this structure might arise from gallium telluride phonons. We also observed similar structures on $0.35 \mathrm{~J} / \mathrm{cm}^{2}, 1$ pulse samples. For this contacts traces of gallium telluride have also been observed by Mössbauer spectroscopy ${ }^{10}$ and XRD. ${ }^{21}$ Beside gallium telluride phonons additional phonons are detected at 195 and $253 \mathrm{~cm}^{-1}$ [Fig. 5(b)] by Raman spectroscopy. These are the $E_{g}$ and $A_{1 g}$ Inodes of elemental As. ${ }^{22}$ This suggests that an exchange reaction between $\mathrm{Te}$ and $\mathrm{As}$ takes place during the annealing process. A gallium telluride phase is formed an elemental As is set free in islands on top of the sample surface. The effect of elemental As to the Schottky barrier height is not yet clear. It is reported that the barrier height could be decreased by outdiffusion of As causing the formation of As vacancies ${ }^{23}$ and increased by the formation of As clusters at the surface. ${ }^{24}$ Even on the ohmic contacts annealed with an energy fluency of $2.5 \mathrm{~J} / \mathrm{cm}^{2}$ and 1 pulse elemental As is detected. From the presence of As in the surface layer of ohmic and of rectifying contacts it can be concluded that the transition to ohmic characteristics is not caused by As. An explanation for the rectifying character even in the presence of gallium telluride can be the inhomogeneity of the contact region and the formation of a compound consisting of $\mathrm{Ga}_{x} \mathrm{Te}_{y}$ because no clear $\mathrm{Ga}_{2} \mathrm{Te}_{3}$ phonon peaks are observed which could also be caused by a highly disordered $\mathrm{Ga}_{2} \mathrm{Te}_{3}$ phase. The increasing background towards lower wave numbers in Fig. 5 is caused by a diffuse backscattering of the laser light at the rough surface of the sample.

On the ohmic laser alloyed samples no features from a gallium telluride compound have been found. Again a peak near the GaAs TO phonon frequency is observed. This peak appears in the forbidden and in the allowed scattering configuration. Additionally no $\Omega^{+}$mode is found up to $2000 \mathrm{~cm}^{-1}$ relative to the laser line. Therefore, from Raman measurements a doping of the contact region of $10^{17}$ to $10^{20} \mathrm{~cm}^{-3}$ can be excluded. Again an explanation of the observed peak by an $\Omega^{-}$mode can be excluded and it should be a defect or disorder activated TO phonon. Combining this with Mössbauer results, a doping of the contact region larger than $10^{17} \mathrm{~cm}^{-3}$ can be ruled out. Also a small LO phonon is observed which is shifted towards 286 $\mathrm{cm}^{-1}$. Such a shift has been reported for ion bombarded GaAs (100) wafers and has been explained by the change in the ionic plasma frequency-changing the TO/LO splitting-due to a high density of defects. ${ }^{25}$ Preliminary photoluminescence experiments also indicate the formation of a disordered GaAs surface layer.

An additional feature obscrved by Raman spectroscopy corresponds to amorphous-like GaAs [Fig. 5(c)]. This spectrum represents rather the density of phonon states than a certain phonon peak.

In the Raman spectra an increasing background towards higher wave numbers [Fig. 5(b)] is observed. This belongs to a very broad structure (Fig. 6), which is similar to the one reported previously for furnace alloyed $\mathrm{Au} / \mathrm{Ge}$ contacts to GaAs. ${ }^{26}$ There it has been attributed to a Raman signal of an overdoped compensated sample. Based on this observation, conduction in this contact structure was argued to occur by an impurity assisted resonant tunneling process. We found that the broad structure is not a special feature of ohmic samples. It has been observed on nonohmic samples with even higher intensities. Hence a discussion concerning the ohmic behavior based on this broad structure is not crucial. Additionally the maximum of this peak shifts as a function of the exciting wavelength $\lambda_{L}$ (Fig. 6, inset), but for very different $\lambda_{L}$ also the relative position of the maximum to $\lambda_{L}$ changes. The same feature has been observed on $\mathrm{Au} / \mathrm{GaAs}$ (100) laser mixed samples. Hence, we conclude that it is a photoluminescence signal which is also strengthened by missing of an anti- 


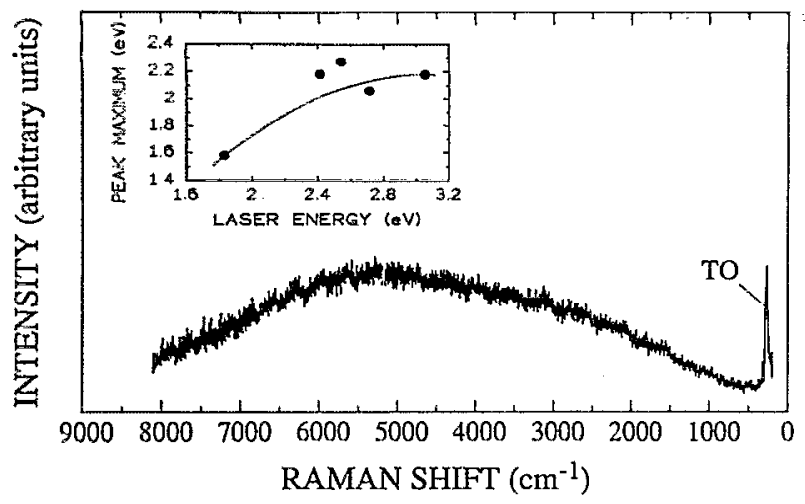

FIG. 6. Raman spectrum $\left(\lambda_{L}=457 \mathrm{~nm}\right)$ of the high-energy shifted region on sample $\left(2.5 \mathrm{~J} / \mathrm{cm}^{2}, 1\right.$ pulse). The maximum of the broad structure shifted as a function of the exciting laser energies (inset).

Stokes signal. The peak maximum seems to depend on the light penetration depth relative to the incorporation length of $\mathrm{Au}$ or of elementary point defects of GaAs.

\section{CONCLUSION}

Comparing the results of the furnace annealed and the laser mixed contacts a different structural nature of the contacts is found. For furnace annealed contacts the mechanism which is responsible for the ohmic behavior is dominated by the formation of $\mathrm{Ga}_{2} \mathrm{Te}_{3}$, polycrystalline $\mathrm{Te}$ and a highly disordered $\mathrm{GaAs}$ layer. $\mathrm{Ga}_{2} \mathrm{Te}_{3}$ is indicated by its vibration modes. No evidence for a doping of the GaAs caused by the furnace annealing is found.

Although on the low-energy fluency laser beam mixed samples gallium telluride compounds are formed, these contacts are highly resistive. On the ohmic laser alloyed contacts a disordered interfacial GaAs layer is present. A doping caused by the mixing can be excluded. Hence, we guess that an electron tunneling through the potential barrier involving localized gap states is the main mechanism for the ohmic behavior as it is also proposed in Ref. 10 .
${ }^{1}$ N. Braslau, J. B. Gunn, and J. C. Staples, Solid State Electron. 10, 381 (1967).

${ }^{2}$ See, for example, G. Robinson, in Physics and Chemistry of III-V Compound Semiconductor Interfaces, edited by C. W. Wilmsen (Plenum, New York, 1985).

${ }^{3}$ E. D. Marshall, B. Zhang, L. C. Wang, P. F. Jiao, W. X. Chen, T. Sawada, S. S. Low, K. L. Kavanagh, and T. F. Kuech, J. Appl. Phys. 62, 942 (1987).

${ }^{4}$ L. C. Wang, X. Z. Wang, S. S. Lau, T. Sands, W. K. Chan, and T. F. Kuech, Appl. Phys. Lett. 56, 2129 (1990).

${ }^{5}$ H. K. Kim, M. Murakami, W. H. Price, and M. Norcott, J. Appl. Phys. 67, 4183 (1990).

${ }^{6}$ R. P. Gupta and W. S. Kohkle, Solid State Electron. 28, 823 (1985).

${ }^{7}$ L. C. Wang, B. Zhang, F. Fang, E. D. Marshall, S. S. Lau, T. Sands, and T. F. Kuech, J. Mater. Res. 3, 922 (1988).

${ }^{8}$ T. Sebestyen, Solid State Electron. 25, 543 (1982).

${ }^{9}$ K. Wuyts, A. Vantomme, R. E. Silverans, M. Van Hove, and M. Van Rossum, Mater. Res. Soc. Symp. Proc. 144, 545 (1989).

${ }^{10}$ K. Wuyts, R. E. Silverans, M. Van Hove, and M. Van Rossum, Mater. Res. Soc. Symp. Proc. 157, 413 (1990).

${ }^{11} \mathrm{~K}$. Wuyts, G. Langouche, H. Vanderstraeten, R. E. Silverans, M. Van Hove, M. Van Rossum, H. Münder, and H. Lüth, Mater. Res. Soc. Symp. Proc. 181, 345 (1990).

${ }^{12}$ K. Wuyts, J. Watté, R. E. Silverans, H. Bender, M. van Hove, and M. van Rossum, J. Vac. Sci. Technol. B 9, 228 (1991).

${ }^{13} \mathrm{G}$. Langouche, H. Bemelmans, and M. van Rossum, Mater. Res. Soc. Symp. Proc. 104, 527 (1988).

${ }^{14} \mathrm{G}$. Abstreiter, M. Cardona, and A. Pinczuk, in Light Scattering in Solids IV, Topics Appl. Phys. 54, edited by M. Cardona and G. Güntherodt (Springer-Verlag, Berlin, 1984).

${ }^{15}$ G. Abstreiter, E. Bauser, A. Fischer, and K. Ploog, Appl. Phys. 16, 345 (1978).

${ }^{16} \mathrm{H}$. Bender (private communication).

${ }^{17}$ K. K. Tiong, P. M. Amirtharaj, F. H. Pollak, and D. E. Aspnes, Appl. Phys. Lett. 44, 122 (1984).

${ }^{18}$ R. P. Sharma, R. Bhadra, L. E. Rehn, P. M. Baldo, and M. Grimsditch, J. Appl. Phys. 66, 152 (1989).

${ }^{19} \mathrm{~K}$. Wuyts, J. Watté, H. Vanderstraeten, H. Pattyn, G. Langouche, R. E. Silverans, H. Münder, M. G. Berger, H. Lüth, G. Zegbe, J. C. Jumas, M. van Hove, H. Bender, G. Borghs, and M. van Rossum, Nuclear Instrum. Methods $\mathbf{B}$ (to be published).

${ }^{20}$ M. H. Brodsky, in Light Scattering in Solids I, Topics Appl. Phys. 8, edited by M. Cardona (Springer-Verlag, Berlin, 1984).

${ }^{21} \mathrm{H}$. Vanderstraeten (private communication).

${ }^{22}$ W. Richter, T. Fjeldy, J. Renucci, and M. Cardona, in Lattice Dynamics, edited by M. Balkanski (Flammerion Sciences, Paris, 1978).

${ }^{23}$ T. Zhang, T. W. Sigmon, K. H. Weiner, and P. G. Carey, Appl. Phys. Lett. 55, 580 (1989).

${ }^{24}$ M. L. Kniffin and C. R. Helms, J. Appl. Phys. 68, 1367 (1990).

${ }^{25}$ G. Burns, F. H. Dacol, C. R. Wie, E. Burstein, and M. Cardona, Solid State Commun. 62, 449 (1987).

${ }^{26}$ D. Kirillov and Y. Chung, Appl. Phys. Lett. 51, 846 (1987). 
Journal of Applied Physics is copyrighted by the American Institute of Physics (AIP). Redistribution of journal material is subject to the AIP online journal license and/or AIP copyright. For more information, see http://ojps.aip.org/japo/japcr/jsp

Copyright of Journal of Applied Physics is the property of American Institute of Physics and its content may not be copied or emailed to multiple sites or posted to a listserv without the copyright holder's express written permission. However, users may print, download, or email articles for individual use. 